\title{
Research Article \\ Effect of Different Gamma Dose and Chemical Etching on Pre- and Post-Alpha-Irradiated PM-355 Polymer
}

\author{
Mostafizur Rahaman $(\mathbb{D}$, Govindasami Periyasami $(\mathbb{D}$, and Ali Aldalbahi \\ Department of Chemistry, College of Science, King Saud University, Riyadh 11451, Saudi Arabia \\ Correspondence should be addressed to Mostafizur Rahaman; mrahaman1997@gmail.com \\ and Ali Aldalbahi; aaldalbahi@ksu.edu.sa
}

Received 22 September 2020; Revised 29 January 2021; Accepted 9 February 2021; Published 18 February 2021

Academic Editor: Cornelia Vasile

Copyright (C) 2021 Mostafizur Rahaman et al. This is an open access article distributed under the Creative Commons Attribution License, which permits unrestricted use, distribution, and reproduction in any medium, provided the original work is properly cited.

\begin{abstract}
This work is based on the effect of different gamma doses with pre- and post-alpha-irradiated PM-355 polymer (polycarbonate of allyl diglycol). The phase crystallinity and structural analysis of the reference and irradiated PM-355 polymer were analyzed using an X-ray diffraction (XRD) study. It is revealed that the irradiation and etching reduce the \%crystallinity but increase the crystallite size of the PM-355 polymer. The increase in crystallite size of PM-355 polymer after irradiation is supported by the scanning electron microscopic (SEM) analysis. The etching of the samples results in an increase in its track diameter. The optical band gap energy, measured by ultraviolet-visible (UV-VIS) spectroscopy, shows a decrement trend with the increase of gamma and alpha irradiation doses, and etching for all sets of samples under investigation. The number of carbon atoms per conjugation and per cluster has increased after gamma irradiation and etching. However, the increment is more pronounced for etched samples compared to nonetched ones. This indicates that etching results in a bigger size of cluster. Photoluminescence (PL) for both cases before and after etching has a dominant peak around $430 \mathrm{~nm}$ before and after irradiation, and change in peak intensity after irradiation confirmed that particle bombardment induced defects and clusters in the PM-355, which serves as nonradiative centers. The polymer can be used as a detector for gamma irradiation.
\end{abstract}

\section{Introduction}

The damage within the materials happened by many means: radiation is one of them. When the radiation of heavily charged particles is incident on a solid material, then tracks are formed within it [1]. Silk and Barnes reported the formation of tracks on mica film [2]. In this work, the tracks were created by exposing the mica films on the radiation path of uranium's fission fragments. The track of diameter $300 \AA$ and length of $4.0 \mu \mathrm{m}$ were observed. Benton and Nix reported that there is a certain critical radiation energy dose above which the tracks can be formed within the material and this is dependent on material's characteristics and etching methodology [3]. The heavy charged particle can be detected through the formation of tracks when it falls on targeted insulating polymeric materials known as solid-state nuclear track detector (SSNTD). This technique is widely and successfully used in nuclear, space, and archeological sciences and technologies to understand the mechanism of track formation within the targeted solid materials. Fleischer et al. have investigated the radiation effect of heavy charged particles when passing through the insulating target $[4,5]$. They reported the formation of tracks within the insulating material because of ionization of positively charged particles. A model was proposed, namely, ion explosion spike, which stated that the ejection of particles was because of mutual repulsion among the positively charged ions and this was in agreement with the experimental results as concluded therein.

Some commonly used materials for SSNTD are poly allyl diglycol carbonate (PADC), known as CR-39, polyethylene terephthalate (PET), Makrofol-KG (a bisphenol a polycarbonate), and super grad PM-355. The CR-39 is transparent for the visible region of light and is absolutely opaque in 
the UV range. It is for the detection of cosmic ray, heavy ion collision studies and to search for magnetic monopoles and for radon and neutron dosimetry.

In another study, the effect of He-Ne laser ray was investigated on CN-85 (cellulose nitrate) and CR-39 polymers based SSNTDs [6]. Before laser irradiation, both polymers were bombarded with italic $>$ /italic $>$-particle of energy $5.44 \mathrm{MeV}$ using a current source of $241 \mathrm{Am}$. The energy density of the laser-irradiated beam was varied up to $217 \mathrm{~J} / \mathrm{cm}^{2}$. They investigated the optical property of both polymers using PL and UV-VIS spectra. It was reported that the laser-irradiated polymers showed the highest value of optical absorption compared to the $\alpha$-bombarded and neat polymers. The absorption was shifted to a longer wavelength when the dose of laser radiation was increased. Moreover, the optical band gap energy and the intensity of PL spectra of the test samples were decreased as the energy density of laser radiation was increased. This can be attributed to the scission of polymer chains because of laser irradiation. It was concluded that a good correlation between the test results and the applied doses were observed. Recently, Chan and his coworkers investigated the effect of $\mathrm{NaOH} /$ ethanol etchant on the CR-39 polymer [7]. It was shown that the bulk etched property of the detector was increased with the increase in molarity of etchant and followed by stirring. In addition, when the track itched property was considered, the length of track was shortened after treatment of polymer with the etchant. Studies on the SSNTD, namely, CR-39 was made by various authors in the past and reported in the literature [8-10].

The PM-355 is a SSNTD and has the same chemical formula that of CR-39 or PADC. PM-355 SSNTDs have been widely used for the detection of ions [11-15]. Nouh and Bahammam have studied the effect of electron beam irradiation on PM-355 polymer within the radiation dose 20$250 \mathrm{kGy}$ [13]. They reported the hardness value of the nonirradiated and irradiated samples with respect to applied load and radiation doses. The hardness value of the base polymer was approximately 4.2 $\mathrm{MPa}$ at $25 \mathrm{mN}$ load for nonirradiated sample. This value had increased up to approximately 10.8 MPa with the increase in applied measurement load at $700 \mathrm{mN}$. On the contrary, with the increase in radiation dose, initially, the hardness value was decreased up to $170 \mathrm{kGy}$ dose and then increased up to $250 \mathrm{kGy}$ dose because of crosslinking of the polymer but it always showed less value compared to the base polymer. In another study, Nouh et al. have exposed the PM-355 to X-ray radiation from a $50 \mathrm{kV}$ X-ray tube where the dose range was $10-300 \mathrm{kGy}$ [15]. The results showed that the chain scission of PM-355 polymer occurred within the radiation dose range $10-20 \mathrm{kGy}$. It generated the free radicals when irradiated within the $20-100 \mathrm{kGy}$ range. This generation of free radicals resulted in cross-linking within the polymer matrix and consequently increased the hardness and refractive index values. In the range of radiation dose $100-300 \mathrm{kGy}$, the PM-355 leads to degradation as proved by FTIR spectral analysis where the degree of ordering was enhanced as supported by XRD analysis and hence both the hardness and refractive index were decreased. The polycarbonates, for example, Makrofol are very sensitive for charged particles (protons, electron, and alpha particles), gamma rays, and $\mathrm{X}$-rays. Therefore, they present a very suitable approach for detecting heavy ions in the understanding of cosmic rays, nuclear reactions, and investigation of heavy elements, which are most appropriate for microfilter technology. The Makrofol-DE has also been used for ion track detection and to make the track-etched membranes and used as a microfilter [16]. In this work, the polymer was irradiated with gamma rays, which was emitted from ${ }^{60} \mathrm{Co}$. The energy of the radiation was $1.25 \mathrm{MeV}$. The measurement was carried out within the radiation doses $20-1000 \mathrm{kGy}$. Optical properties and hardness of the samples were tested. UV-VIS spectrum analysis reported the decrement in band gap energy after irradiation with gamma rays. The carbon atom number was also increased within the irradiated samples compared to base polymer. PL peak intensity was also reduced after irradiation. This was because of the formation of defects within the irradiated samples. The hardness value was significantly increased after irradiation with gamma doses. It was reported 4.78 MPa for the base polymer and 23.67 MPa for the highest dose irradiated sample. Contact angle measurement showed an increase in wettability after gamma irradiation.

The obtaining of a high value of track diameter after irradiation on polymer implies its effectiveness as a good detector. This we may achieve by varying radiation type, and irradiation, etching, and measurement conditions. Hence, the importance of this study is that we have tested the PM355 detector by varying different doses of gamma irradiation, gamma followed by alpha irradiation, alpha followed by gamma irradiation, and finally followed by etching for getting high track diameter value. The samples are tested through XRD, SEM, UV-VIS, and photoluminescence spectra. An attempt has been made to explain the results on the basis of structural changes that have happened inside the polymer because of irradiation and etching.

\section{Material, Methods, and Experimental}

2.1. Material and Sample Preparation. In this work, the SSNTD that we used was PM-355. It had thickness of 500 micron and density of $1.32 \mathrm{~g} / \mathrm{cm}^{3}$. The sample was prepared by molding method (Pershore Moulding Limited, England). The test samples were divided into three sets, each set containing 5 detectors. The samples were initially irradiated only with gamma dose and then followed by pre- and postalpha irradiation with different gamma dose as shown in Table 1.

2.2. Gamma Irradiation. For gamma irradiation, we used the $\mathrm{Co}^{60}$ source with half-life of 5.2714 years. This source radiates the energy of 1.17 and $1.33 \mathrm{MeV}$. The specimens were gamma irradiated with different doses of 20,40,60, 80, and $100 \mathrm{kGy}$.

2.3. Alpha Irradiation. The samples were alpha irradiated using a 0.1 micro $\mathrm{Ci}^{241} \mathrm{Am}$ source, which emitted radiant energy of $5.49 \mathrm{MeV}$. The specimens were alpha irradiated in air for 5 seconds (time was measured using a stopwatch) by keeping a distance of $2 \mathrm{~cm}$ apart from the irradiation source. 
TABLE 1: Gamma irradiated sample details.

\begin{tabular}{|c|c|c|c|c|c|}
\hline \multirow{2}{*}{$\begin{array}{l}\text { Sample } \\
\text { name }\end{array}$} & \multirow{2}{*}{$\begin{array}{l}\text { Set I (only } \\
\text { gamma } \\
\text { irradiation) } \\
\text { Gamma } \\
\text { dose (kGy) }\end{array}$} & \multicolumn{2}{|c|}{$\begin{array}{l}\text { Set II (gamma } \\
\quad+\text { alpha) }\end{array}$} & \multicolumn{2}{|c|}{$\begin{array}{l}\text { Set III (alpha } \\
\text { +gamma) }\end{array}$} \\
\hline & & $\begin{array}{c}\text { Gamma } \\
\text { dose } \\
(\mathrm{kGy})\end{array}$ & $\begin{array}{l}\text { Alpha } \\
\text { radiation } \\
\text { time }(s)\end{array}$ & $\begin{array}{c}\text { Alpha } \\
\text { radiation } \\
\text { time }(\mathrm{s})\end{array}$ & $\begin{array}{c}\text { Gamma } \\
\text { dose } \\
(\mathrm{kGy})\end{array}$ \\
\hline SGR & 0 & 0 & 5 & 5 & 0 \\
\hline SG1 & 20 & 20 & 5 & 5 & 20 \\
\hline SG2 & 40 & 40 & 5 & 5 & 40 \\
\hline SG3 & 60 & 60 & 5 & 5 & 60 \\
\hline SG4 & 80 & 80 & 5 & 5 & 80 \\
\hline SG5 & 100 & 100 & 5 & 5 & 100 \\
\hline
\end{tabular}

2.4. Chemical Etching. The chemical etching was performed for sample sets 2 and 3 to observe the nature of tracks. These samples were etched in $\mathrm{NaOH}$ solution $(6.25 \mathrm{~N})$ at temperature $70^{\circ} \mathrm{C}$ for 3 hours.

2.5. X-Ray Diffraction (XRD) Studies. The phase crystallinity and structural analysis of the test specimens were performed through XRD analysis (X'pert PRO; PANanalytical). The measurement was carried out at room temperature by applying the radiation of wavelength $1.54 \AA$ emitted from the $\mathrm{Cu}-$ $\mathrm{K}_{\alpha}$ source.

2.6. Field Emission Scanning Electron Microscopy (FESEM). The surface morphology of pre- and postirradiated specimens was observed by FESEM (JEOL FESEM). Most of the specimens were tested at $10-30 \mathrm{keV}$, whereas the insulating samples were tested at low energy means within several hundred $\mathrm{eV}$.

2.7. Optical Microscope. The track diameter was measured after etching of PM-355 SSNTDs using an optical microscope (Ziess, Germany). The magnification of the measurement was $400 x$.

2.8. Photoluminescence (PL). It is used for finding the defects in semiconductor materials. This is mostly used for determining the shallow-level defects, however, is also applicable to determine deep-level defects, depending on the radiative recombination. The PL spectra of the specimens were recorded using a JASCO FP-8200 Spectrofluorometer. The measurement was performed at ambient temperature where the excitation wavelength was $250 \mathrm{~nm}$.

2.9. UV-VIS Spectroscopy. The spectra of the test specimens were recorded within the wavelength range of $200-800 \mathrm{~nm}$. The test was performed at ambient temperature using the instrument JASCO V-670 UV/VIS Spectrophotometer.

\section{Results and Discussion}

3.1. X-Ray Diffraction (XRD) Study. Figure 1 shows the XRD spectra of reference, only gamma irradiated, and alpha plus gamma with chemically etched PM-355 SSNTDs within the $2 \theta$ (Bragg angle) value 10 to $80^{\circ}$. A large and broad peak at

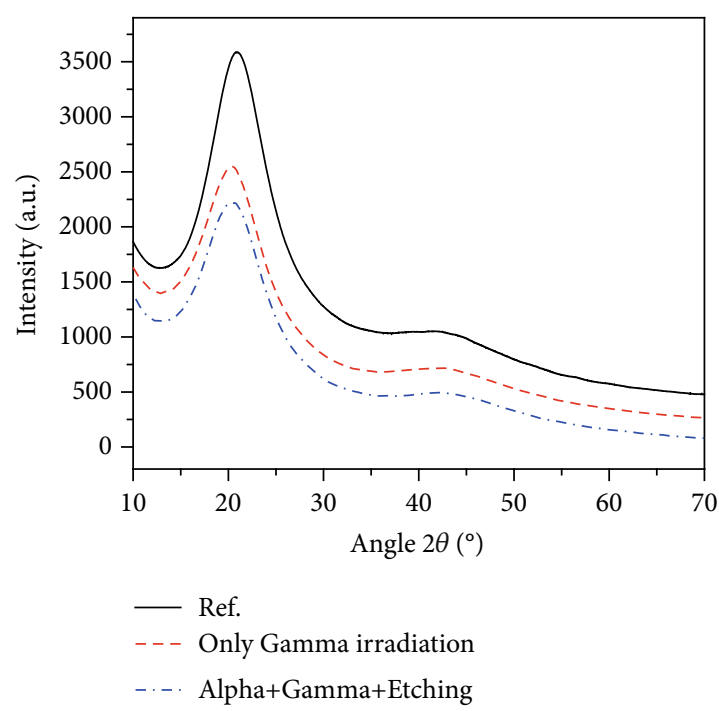

FIGURE 1: XRD spectra of reference, gamma irradiated, and irradiated plus chemically etched PM-355 SSNTD.

$2 \theta=21.29^{\circ}$ is noticed for the reference sample. This indicates that the PM-355 polymer is mostly dominated by an amorphous phase and hence is partly crystalline in nature. The peak is shifted to $20.36^{\circ}$ for gamma-irradiated sample and 20.29 for chemically etched PM-355. The shifting of the peak position may be due to the stress produced because of the irradiation of the PM-355 SSNTDs. A quantitative estimation of the XRD parameters like \%crystallinity $\left(\% X_{c}\right)$, peak maxima, full with at half maxima (FWHM, denoted as $b$ ), interplanar distance ( $d$-spacing), interchain distance $(r)$, crystallite size $(L)$, microstrain $(\varepsilon)$, and distortion parameter $(g)$ has been made using the following equations [17]:

$$
\begin{aligned}
\% X_{C} & =\left(\frac{A_{C}}{A_{C}+A_{A}}\right) \times 100 \%, \\
L & =\frac{K \lambda}{b \cos \theta}, \\
d & =\frac{\lambda}{2 \operatorname{Sin} \theta}, \\
r & =\frac{5 \lambda}{8 \operatorname{Sin} \theta}, \\
\varepsilon & =\frac{b \operatorname{Cos} \theta}{4}, \\
g & =\frac{b}{\tan \theta},
\end{aligned}
$$

where $A_{C}$ is the area of crystalline phase under the curve, $A_{A}$ is the area of amorphous phase under the curve, $K$ is the Scheller constant (has been taken as 0.9 ), and $\lambda$ is the wavelength of X-ray radiation (1.54 $\AA$ ). In the above equations, $\theta$ has been taken in radian. The calculated parameters based on the plots and above equations are presented in Table 2. It is seen from the table that the crystallinity $\left(\% X_{c}\right)$ of PM355 polymer has decreased after both the gamma irradiation 
TABLE 2: Parameters extracted from XRD analysis.

\begin{tabular}{lcccccccc}
\hline Samples & Peak $(2 \theta)$ & $b(\theta)$ & $\mathrm{d}(\AA)$ & $\varepsilon$ & $g(\%)$ & $r(\AA)$ & $\% X_{c}$ & $L(\AA)$ \\
\hline Reference & 21.29 & 6.23 & 4.17 & 1.53 & 33.13 & 5.21 & $28.4 \%$ & 12.96 \\
Ref+gamma & 20.36 & 6.08 & 4.35 & 1.50 & 33.85 & 5.44 & $22.5 \%$ & 13.26 \\
Ref+alpha+gamma+etching & 20.24 & 6.05 & 4.38 & 1.49 & 33.85 & 5.48 & $21.6 \%$ & 13.33 \\
\hline
\end{tabular}

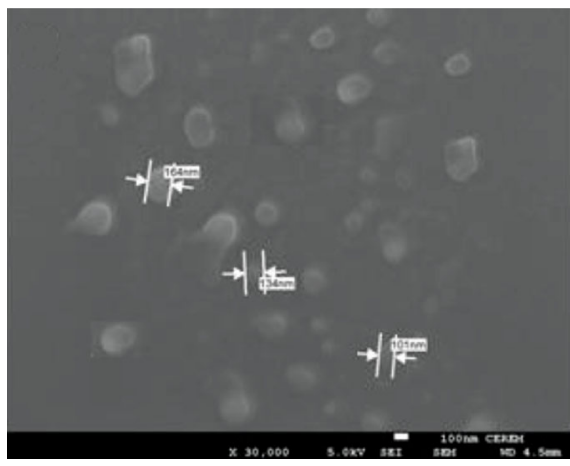

(a)

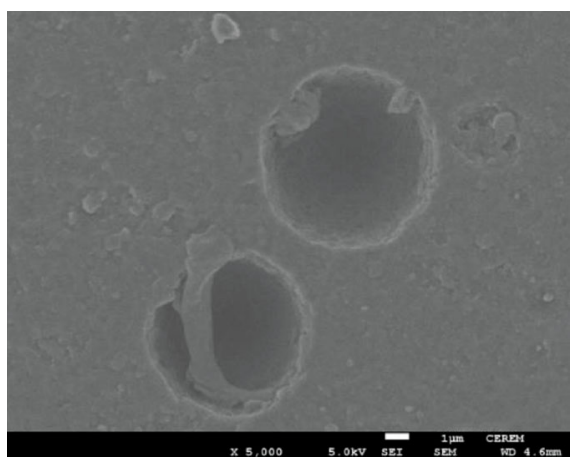

(c)

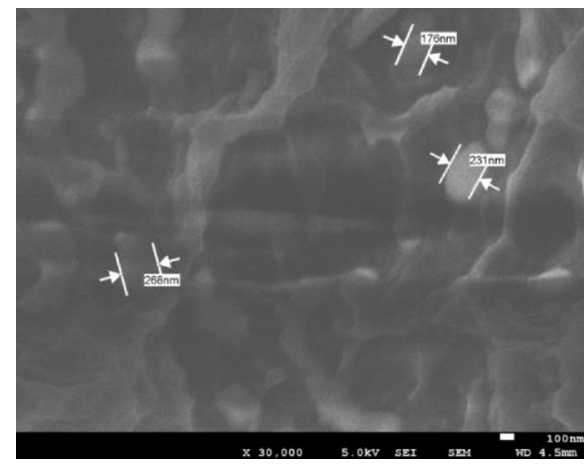

(b)

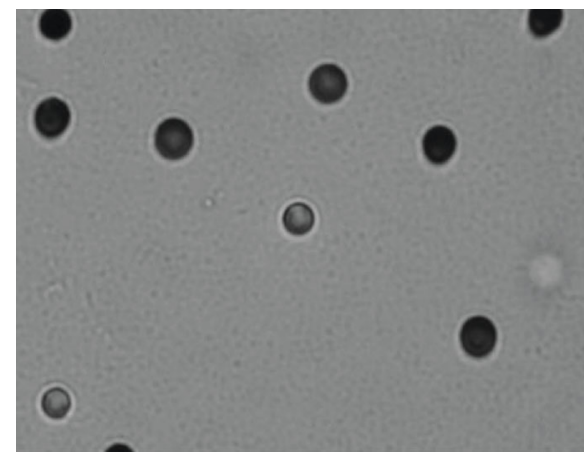

(d)

FIGURE 2: (a) SEM image of reference. (b) SEM image of only gamma-irradiated PM-355. (c) SEM image of gamma and alpha irradiated +chemically etched PM-355. (d) Microscopic image of alpha track.

and chemical etching. Actually, the irradiation causes the cross-linking inside the polymer by making new bonds among the neighboring molecular chains. This results the change in regularly ordered crystallites into nonordered ones and hence decrease in crystallinity [18]. Both the figure and table show that the FWHM (b) of PM-355 peak become narrow after gamma irradiation and chemical etching. This indicates that the crystallite size $(L)$ has increased after the gamma irradiation and chemical etching as can be seen from the table. The value of FWHM is less, but the value of crystallite size is higher for our study compared to the study mentioned in literature [18]. The interplanar $(d)$ and interchain $(r)$ distances have increased after irradiation as seen from the table. This is due to the change in some ordered crystalline phase into amorphous one, which increases the disorderness that means the distortion parameter $(g)$, and thereby the increase in interplanar and interchain distances [18]. It is also seen from the table that the parameter, microstrain $(\varepsilon)$, decreases after irradiation of PM-355 polymer. This can be attributed to the mismatching of atoms or ions [18].
3.2. Morphology through Scanning Electron Microscope (SEM). In Figures 2(a)-2(c), we have presented the morphological diagram of reference PM-355, only gamma radiated, and alpha plus gamma radiated with chemically etched samples photographed by a scanning electron microscope (SEM), respectively, and Figure 2(d) alpha track image photographed by an ordinary microscope of the PM-355 SSNTD. Figure 2(a) shows that the grains of reference SSNTDs possess a spherical-like geometrical shape within the diameter range of $100-170 \mathrm{~nm}$, which was further increased in size after irradiated with gamma-ray as observed in Figure 2(b). The surface of gamma-irradiated sample also becomes more rough compared to the reference one. This result of the SEM study is in accordance with the XRD finding, which meant that the crystallite size of polymer has increased after the application gamma radiation. However, there is a distinct difference between the size of crystallite and grain. The size of the grain is much more higher compared to the size of crystallite. This indicates that the grain is formed by the accumulation of several crystallites. Figures 2(c) and 2(d) are the images of 
alpha plus gamma irradiated with chemically etched samples photographed by a SEM and ordinary microscope, respectively, which show circular-shaped nuclear tracks due to alpha radiation with an average diameter of $8.75 \mu \mathrm{m}$.

3.3. UV-VIS Spectroscopy. The absorbance spectra of gamma irradiation samples as a function of different dose (set 1), postalpha irradiation with different gamma dose (set 2), and prealpha irradiation with different gamma dose (set 3) within the range of $225-350 \mathrm{~nm}$ are depicted in Figure 3, respectively. It is observed from the figure that the absorption edge initially shifted sharply to the longer wavelength and then very small shifting has been noticed with further increment in radiation dose. The shifting of absorption edge to higher wavelength region indicates the decrement in optical band gap energy [19-23]. Actually, with the increase in gamma dose, there is the scission of bonds within the molecular chains with the formation of free radicals that is finally cross-linked to form some new bonds and results in the shifting of absorption edge [18-20,24]. The absorption edge is resulting because of $\pi-\pi *$ transition, where for excitation of $\pi$-electrons require less energy and consequently, the transition occurs at a longer wavelength [21].

The effect of etching on the absorbance of sample set 2 and set 3 is investigated and presented in Figures 4(a) and $4(\mathrm{~b})$, respectively. It is observed that the variation in absorbance follows the same trend as it was before etching but the relative absorbance increases. This increase in absorbance value indicates that the etching helps in the production of some free radicals within the sample during irradiation. There is also a little bit of shift of absorption edge towards the higher wavelength. This is attributed to the formation of some new chemical species within the polymer detector [23]. Comparing the absorbance before and after etching, it can be inferred that etching makes some structural changes within the material and increases the track diameter.

The band gap energy of a material can be calculated through the measurement of optical absorption spectra. To determine the optical band gap energy, the data from the UV-VIS optical absorption spectra were used for gamma irradiated and pre- and post-alpha-irradiated samples as shown in Figures 3 and 4. Initially, we calculate the value of absorption coefficient $\alpha$ for the estimation of band gap energy. The value of $\alpha$ was calculated using the absorbance data as follows:

$$
\alpha(\lambda)=2.30 A t^{-1},
$$

where $t$ indicates the specimen thickness and $A$ is absorbance [1]. The value of $A$ depends on the photon energy as follows $[1,3]$ :

$$
(\alpha h v)^{2}=A\left(h v-E_{g}\right)^{m}
$$

In the above equation, the power factor $m$ is a constant quantity, which determines the nature of optical transition. The values of $m$ are $1 / 2,3 / 2,2$, and 3 for direct allowed, direct forbidden, indirect allowed, and indirect forbidden transitions, respectively. $E_{g}$ represents the band

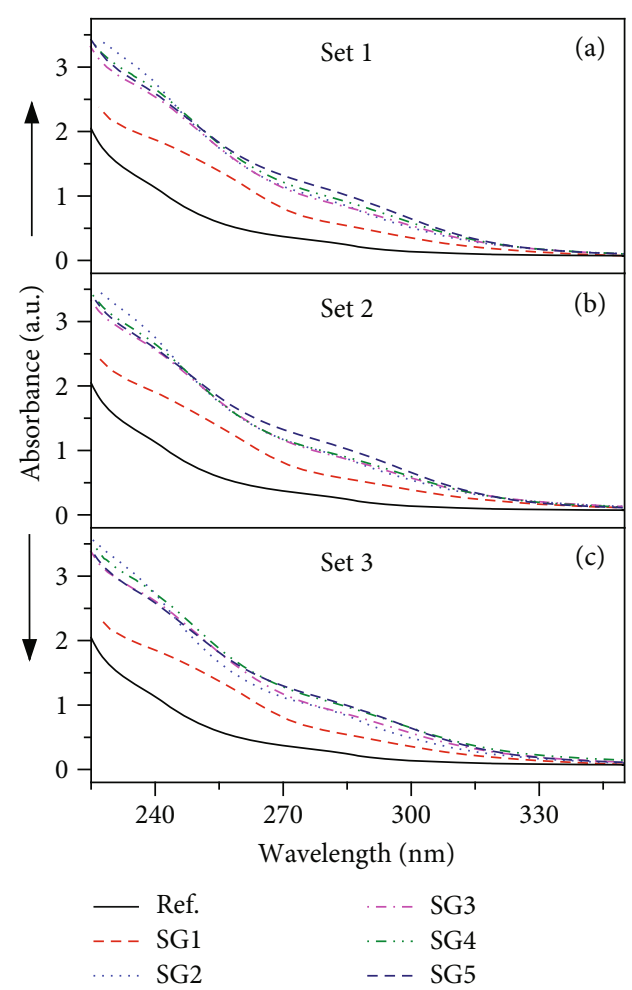

Figure 3: Absorption spectra (a) for different gamma dose irradiated, (b) for postalpha irradiation with different gamma dose, and (c) for prealpha irradiation with different gamma dose of PM-533 SSNTDs.

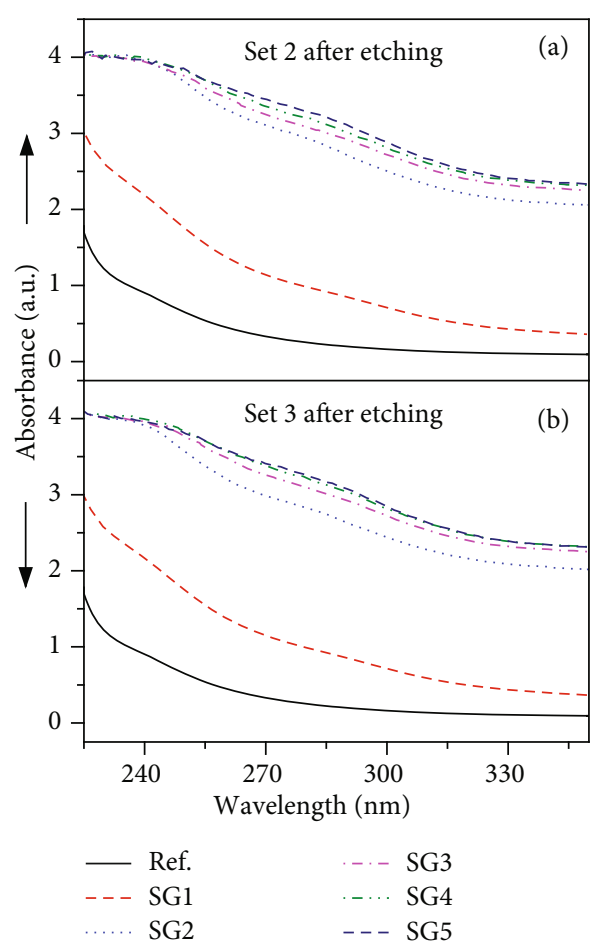

FIgURe 4: Absorption spectra of (a) set 2 and (b) set 3 after etching at $70^{\circ} \mathrm{C}$ for $3 \mathrm{hrs}$. 
TABLE 3: Band gap energy for all sets at different gamma doses of PM-355 SSNTDs.

\begin{tabular}{|c|c|c|c|c|c|c|c|c|}
\hline \multirow{2}{*}{ Sample name } & \multirow{2}{*}{ Gamma dose (kGy) } & \multicolumn{5}{|c|}{ Band gap energy $(\mathrm{eV})$} & \multicolumn{2}{|c|}{$\begin{array}{c}\text { Track diameter } \\
(\mu \mathrm{m})\end{array}$} \\
\hline & & Set 1 & Set 2 & Set 3 & Set 2 after etching & Set 3 after etching & Set 2 & Set 3 \\
\hline SGR & 0 & 5.50 & 5.50 & 5.50 & 5.46 & 5.54 & 5.63 & 5.63 \\
\hline SG1 & 20 & 5.42 & 5.44 & 5.47 & 5.29 & 5.38 & 8.75 & 10.00 \\
\hline SG2 & 40 & 5.23 & 5.26 & 5.27 & 4.18 & 4.27 & - & - \\
\hline SG3 & 60 & 5.14 & 5.16 & 5.19 & 4.00 & 4.12 & - & - \\
\hline SG4 & 80 & 5.12 & 5.13 & 5.15 & 3.83 & 3.95 & - & - \\
\hline SG5 & 100 & 5.06 & 5.09 & 5.11 & 3.71 & 3.82 & - & - \\
\hline
\end{tabular}

-: irregular shape and very big size tracks.

TABLE 4: The calculated value of $N$ and $M$.

\begin{tabular}{|c|c|c|c|c|c|c|c|c|c|c|c|}
\hline \multirow{3}{*}{ Sample name } & \multirow{3}{*}{ Gamma dose (kGy) } & \multicolumn{10}{|c|}{ Carbon atom number } \\
\hline & & \multicolumn{2}{|c|}{ Set 1} & \multicolumn{2}{|c|}{ Set 2} & \multicolumn{2}{|c|}{ Set 3} & \multicolumn{2}{|c|}{$\begin{array}{l}\text { Set } 2 \text { after } \\
\text { etching }\end{array}$} & \multicolumn{2}{|c|}{$\begin{array}{c}\text { Set } 3 \text { after } \\
\text { etching }\end{array}$} \\
\hline & & $N$ & $M$ & $N$ & $M$ & $N$ & $M$ & $N$ & $M$ & $N$ & $M$ \\
\hline SGR & 0 & $\sim 3$ & 38.9 & $\sim 3$ & 38.9 & $\sim 3$ & 38.9 & $\sim 3$ & 39.5 & $\sim 3$ & 39.7 \\
\hline SG1 & 20 & $\sim 3$ & 40.1 & $\sim 3$ & 40.8 & $\sim 3$ & 41.2 & $\sim 3$ & 42.1 & $\sim 3$ & 42.4 \\
\hline SG2 & 40 & $\sim 3$ & 43.0 & $\sim 3$ & 43.4 & $\sim 3$ & 43.8 & $\sim 4$ & 67.3 & $\sim 4$ & 69.5 \\
\hline SG3 & 60 & $\sim 4$ & 44.5 & $\sim 4$ & 44.7 & $\sim 4$ & 44.9 & $\sim 5$ & 73.5 & $\sim 5$ & 81.1 \\
\hline SG4 & 80 & $\sim 4$ & 44.9 & $\sim 4$ & 45.1 & $\sim 4$ & 45.6 & $\sim 5$ & 80.2 & $\sim 5$ & 88.3 \\
\hline SG5 & 100 & $\sim 4$ & 46.0 & $\sim 4$ & 47.1 & $\sim 4$ & 47.8 & $\sim 5$ & 85.5 & $\sim 5$ & 90.3 \\
\hline
\end{tabular}

gap energy, whereas the other terms have their usual meaning.

The calculated band gap energy with different gamma doses for nonetched and etched samples are tabulated in Table 3. It is seen from the table that the band gap energy is affected by the application of radiation dose. It is gradually decreasing for all sets under investigation with the increase in radiation dose. This decrement in band gap energy is because of the defects generated inside the detector structure because of gamma irradiation dose [25]. These defects may be due to the creation of conjugated bond because of partial growth of hydrogen gas [25]. The table reveals that the values of band gap energies for etched samples are less compared to their nonetched counterparts. This can be attributed to the fact that etching leads to the breakage of $\mathrm{C}-\mathrm{O}-\mathrm{C}, \mathrm{C}-\mathrm{H}, \mathrm{C}=\mathrm{O}$, and $-\mathrm{C}=\mathrm{C}-$ bonds and hence reduces the band gap energy [25].

The number of carbon atoms per conjugation length $(N)$ for a linear structure is calculated using the following equation [18]:

$$
N=\frac{2 \pi \beta}{E_{g}},
$$

where $\pi=22 / 7$ and $\beta=2.9 \mathrm{eV}$. The term $\beta$ in the above equation expresses the band structure energy of the paired adjacent $\pi$ sites. This is related to the $\pi-\pi *$ transition in $-\mathrm{C}=\mathrm{C}-$ structural bond. The calculated data are presented in Table 4, which reveals that the value of $N$ increases with the increment in radiation dose. Obviously, this increase in the number of carbon atoms in a conjugated system leads to the elimination of hydrogen atom from the $\mathrm{C}-\mathrm{H}$ bond by the gamma irradiation. This is why the value of $E_{g}$ reduces and hence the electronic conduction behavior of polymer is improved with the increase in radiation dose.

The number of carbon atom per cluster $(M)$ can be calculated as [26]

$$
E_{g=34.3 / \sqrt{ } M},
$$

The calculated values of $M$ using the above equation are depicted in Table 4. It is seen from the table that the value of $M$ is increasing with the increase in irradiation dose. This indicated that the cluster/grain size was increased within the polymer after the increment in radiation dose. This finding corroborates with the SEM study that has been mentioned earlier. It is also seen from the table that after etching, the cluster size of etched samples has increased compared to nonetched ones. This is because the energy absorbed due to etching was led to the formation of an extended system of conjugated carbon bond that results in the production of a bigger size of cluster [25].

3.4. Photoluminescence Spectra. The photoluminescence (PL) features of different sets of PM-355 polymer are rarely studied. The PL spectra of nonetched set 1 , set 2 , and set 3 are shown in Figures 5(a)-5(c), respectively. The value of PL peak intensity has been extracted from the figure and plotted in Figure 6 to check their trend in variation with respect to gamma doses. The figure shows that there is a decrement in broadband intensity for all the three sets under investigation 


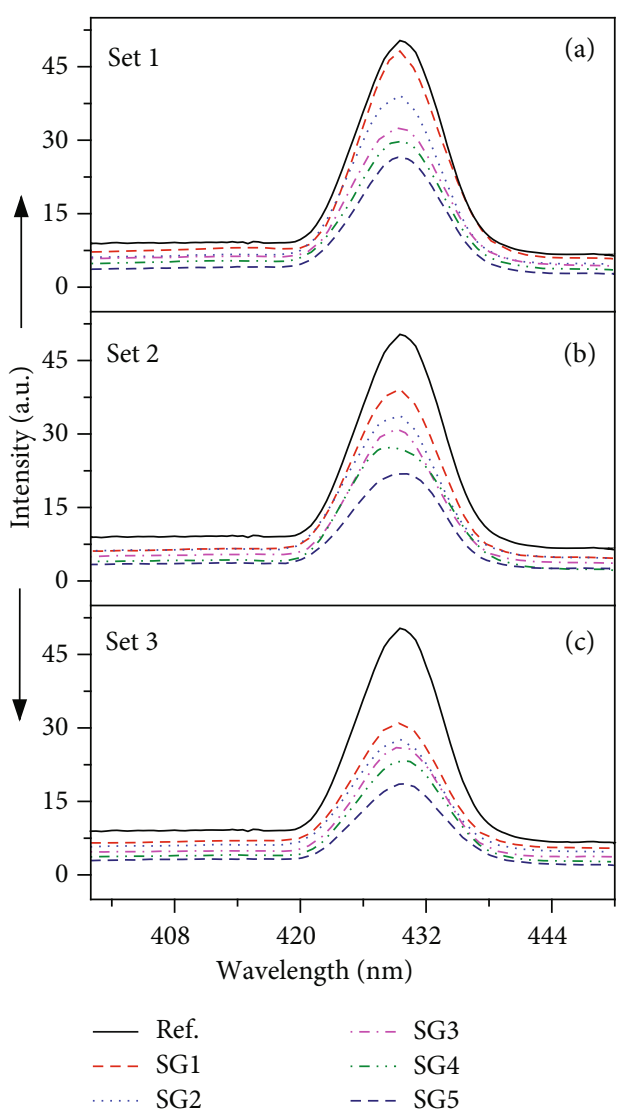

Figure 5: PL spectra (a) for different gamma dose irradiated, (b) for postalpha irradiated with different gamma dose, and (c) for prealpha irradiated with different gamma dose of PM-533 SSNTDs.

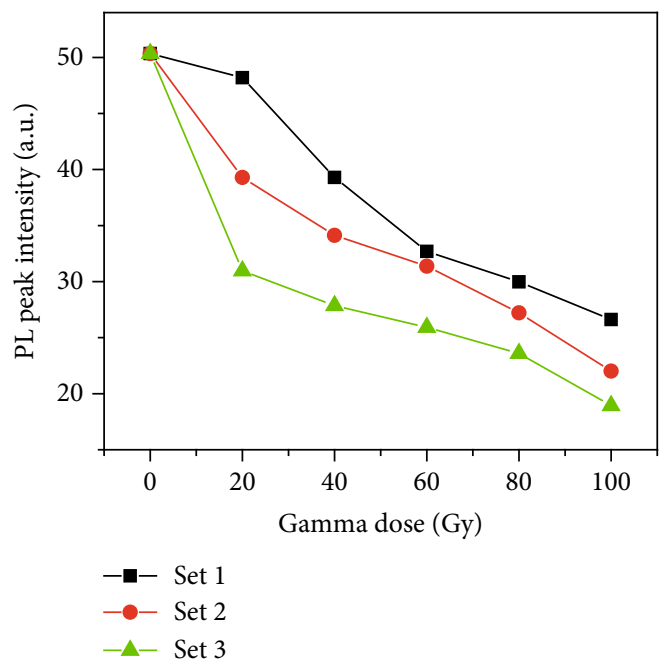

Figure 6: Variation of PL intensity with respect to gamma dose for PM-533 SSNTDs without etching.

when the gamma doses are increased. This decrement in PL intensity can be attributed to the formation of defect after irradiation [20]. Moreover, at a higher dose, the variation of PL intensity is marginal, which may be due to the saturation of polymer with defects. There was the random shifting of

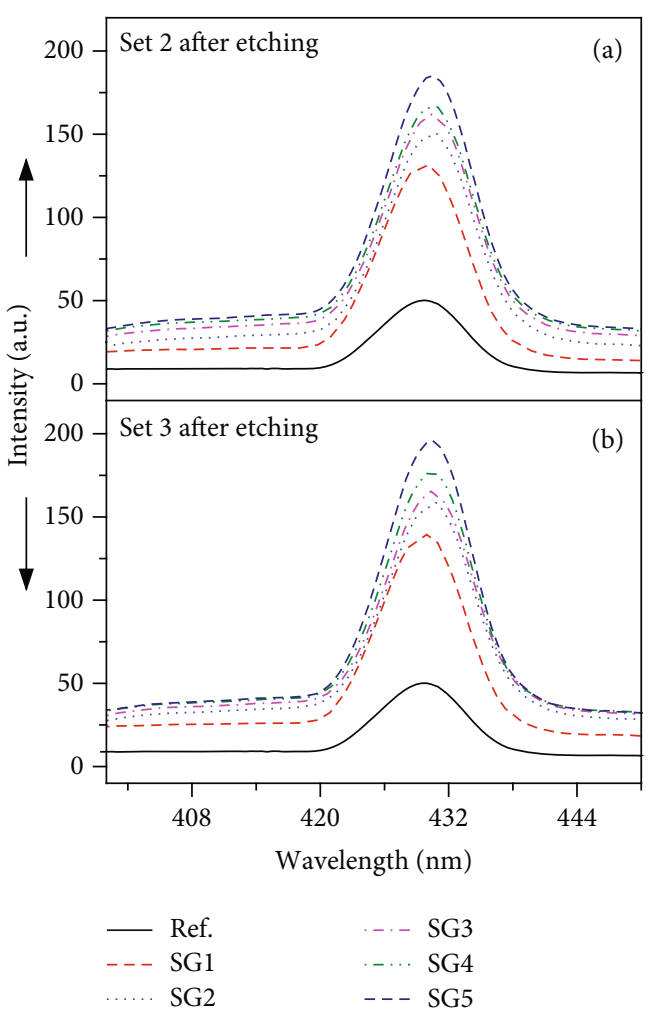

Figure 7: Absorption spectra of (a) set 2 and (b) set 3 after etching at $70^{\circ} \mathrm{C}$ for $3 \mathrm{hrs}$.

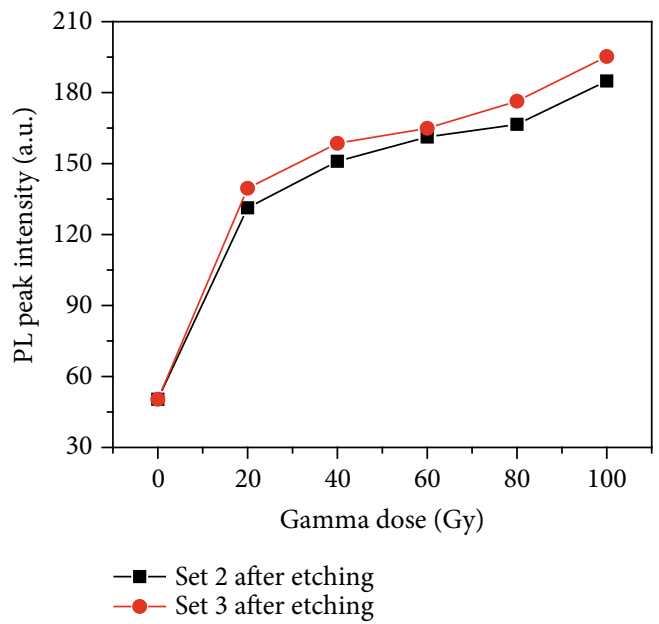

FIgURE 8: Variation of PL intensity with respect to different gamma doses for PM-533 SSNTDs after etching.

peak intensity position towards either shorter or longer wavelength. This is because the irradiation may have resulted in some defect within the polymer and/or may have destructed some chemical species [16].

The photoluminescence spectra of etched samples that are for set 2 and set 3 as a function of gamma doses are presented in (Figures $7(\mathrm{a})$ and $7(\mathrm{~b})$ ). The position of peak intensity is extracted from the figure and is plotted in Figure 8 to check their trend in variation. It is seen that the peak intensity of 
TABLE 5: Variation of track diameters at different gamma doses of chemically etched samples.

\begin{tabular}{|c|c|c|c|}
\hline \multirow[b]{2}{*}{ Sample name } & \multirow[b]{2}{*}{ Gamma dose (kGy) } & \multicolumn{2}{|c|}{ Track diameter $(\mu \mathrm{m})$} \\
\hline & & $\begin{array}{l}\text { Set } 2 \text { after } \\
\text { etching }\end{array}$ & $\begin{array}{c}\text { Set } 3 \text { after } \\
\text { etching }\end{array}$ \\
\hline SGR & 0 & 5.63 & 5.63 \\
\hline SG1 & 20 & 8.75 & 10.00 \\
\hline SG2 & 40 & - & - \\
\hline SG3 & 60 & - & - \\
\hline SG4 & 80 & - & - \\
\hline SG5 & 100 & - & - \\
\hline
\end{tabular}

-: irregular shape and very big size tracks.

etched samples is almost at the same position to that of their nonetched counterpart. The peaks have mostly shifted to longer wavelength compared to reference one. Contrary to the nonetched ones, the peak intensity for etched samples is increasing almost exponentially after the increment in gamma doses as shown in Figure 8. This behavior may be due to etching, which increases the concentration of defect and cluster within the PM-355 polymer that served as the nonradiative centers [4]. Actually, these emission bands are associated with the $\pi *-\pi$ and $\pi *-n$ electronic transitions, which occur within the unsaturated centers of the compounds and account for both increasing and decreasing in the PL intensity [5].

The variation in track diameter with respect to gamma doses for set 2 and set 3 samples after chemical etching is shown in Table 5. It is obvious that at high gamma dose, the tracks become irregular shaped and big in size, which become difficult to measure their size. The size of this track diameter is too high compared to the track diameter mentioned in an earlier study [2].

\section{Conclusions}

In the study of microstructural properties, the X-ray diffraction reveals that the PM-533 polymer is composed of mostly amorphous phase with some crystallinity. The XRD analysis shows that the interplanar distance, interchain distance, distortion parameter, and crystallite size have increased but the microstain and \%crystallinity have decreased after gamma irradiation and etching. The changes in these parameters are due to some stress produced after irradiation and etching. The band gap energy was decreased after the increment in gamma doses. This was evident from the shifting of absorption edges to longer wavelength. However, the rate in decrement of band gap energy is higher for etched samples compared to nonetched ones at a similar gamma exposure dose. It is revealed that the track diameter increases after etching of the samples. The values of $N$ and $M$ have increased both after gamma irradiation and etching. However, the increment is more pronounced in the case of etched samples. The reduction in PL intensity after the increment in gamma doses confirmed the presence of defects and clusters within the PM-355 polymer after bombarding with particle. After etching, the photoluminescence of irradiated samples has an opposite trend in intensity compared to nonetched samples. The size of track diameter of etched samples was very big and was even beyond the measurable range when treated at high radiation doses. This proved that the PM355 polymer can be used as a gamma detector.

\section{Data Availability}

The data used to support the findings of this study are included within this manuscript.

\section{Conflicts of Interest}

There is no competing financial interest among the authors.

\section{Acknowledgments}

The authors would like to extend their sincere appreciation to the Deanship of Scientific Research at King Saud University for funding this work through the research group (RG 1436-005).

\section{References}

[1] H. Kozima, Characteristics of solid-state nuclear track detectors for heavy charged particles-a review, Proceedings of JCF13, Nagoya, Japan, 2013.

[2] E. Silk and R. Barnes, "Examination of fission fragment tracks with an electron microscope," Philosophical Magazine, vol. 4, no. 44, pp. 970-972, 1959.

[3] E. V. Benton and W. D. Nix, "The restricted energy loss criterion for registration of charged particles in plastics," Nuclear Instruments and Methods, vol. 67, no. 2, pp. 343-347, 1969.

[4] R. L. Fleischer, P. B. Price, and R. M. Walker, "Ion explosion spike mechanism for formation of charged-particle tracks in solids," Journal of Applied Physics, vol. 36, no. 11, pp. 36453652, 1965.

[5] N. Bohr, The Penetration of Atomic Particles through Matter, Munksgaard Copenhagen, 1948.

[6] M. Zaki, T. M. Hegazy, and D. H. Taha, "Investigation of the effect of He-Ne laser on the optical properties through etched CN-85 and CR-39 containing alpha tracks," Arab Journal of Nuclear Science and Applications, vol. 46, no. 3, pp. 201-210, 2013.

[7] K. F. Chan, F. M. F. Ng, D. Nikezic, and K. N. Yu, "Bulk and track etch properties of CR-39 SSNTD etched in NaOH/ethanol," Nuclear Instruments and Methods in Physics Research Section B: Beam Interactions with Materials and Atoms, vol. 263, no. 1, pp. 284-289, 2007.

[8] P. A. Mosier-Boss, L. P. G. Forsley, P. Carbonnelle et al., "Comparison of SEM and optical analyses of DT neutron tracks in CR-39 detectors," Radiation Measurements, vol. 47, no. 1, pp. 57-66, 2012.

[9] Z. Tayyeb, "Use of CR-39 polymer for radiation dosimetry," Journal of King Abdulaziz University: Engineering Sciences, vol. 22, no. 1, pp. 79-96, 2010.

[10] M. F. Eissa, "Optical properties of CR-39 track etch detectors irradiated by alpha particles with different energies," Journal of Materials Science and Engineering, vol. 5, no. 1, 2011.

[11] H. A. Al-Jobouri, "Determination the effect of gamma radiation and thermal neutron on PM-355 detector by using FTIR spectroscopy," Detection, vol. 3, no. 3, pp. 15-20, 2015. 
[12] S. A. Nouh, A. A. Naby, and H. M. El Hussieny, "Fast neutron irradiation effects in PM-355 nuclear track detector," Applied Radiation and Isotopes, vol. 65, no. 10, pp. 1173-1178, 2007.

[13] E. H. Aly, "Microstructural investigation of PM-355 nuclear track detector subjected to low-dose gamma irradiation: a positron annihilation lifetime study," Materials Sciences and Applications, vol. 4, no. 10, pp. 622-629, 2013.

[14] S. A. Nouh and S. Bahammam, "Structural and optical investigation of the effect of electron beam irradiation in a PM-355 nuclear track detector," Radiation Effects and Defects in Solids, vol. 167, no. 5, pp. 352-360, 2012.

[15] S. A. Nouh, R. A. Bahareth, and M. M. Abutalib, "X-ray irradiation-induced structural modifications in PM-355 polymeric nuclear track detector film," Radiation Effects and Defects in Solids, vol. 169, no. 6, pp. 499-505, 2014.

[16] A. Tayel, M. F. Zaki, A. B. El Basaty, and T. M. Hegazy, "Modifications induced by gamma irradiation to Makrofol polymer nuclear track detector," Journal of Advanced Research, vol. 6, no. 2, pp. 219-224, 2015.

[17] V. Kumar, Y. Ali, R. G. Sonkawade, and A. S. Dhaliwal, "Effect of gamma irradiation on the properties of plastic bottle sheet," Nuclear Instruments and Methods in Physics Research Section B: Beam Interactions with Materials and Atoms, vol. 287, pp. 10-14, 2012.

[18] V. Kumar, R. G. Sonkawade, and A. S. Dhaliwal, "Gamma irradiation induced chemical and structural modifications in PM355 polymeric nuclear track detector film," Nuclear Instruments and Methods in Physics Research Section B: Beam Interactions with Materials and Atoms, vol. 290, pp. 59-63, 2012.

[19] S. Singh and S. Prasher, "The optical, chemical and spectral response of gamma-irradiated Lexan polymeric track recorder," Radiation Measurements, vol. 40, no. 1, pp. 50-54, 2005.

[20] V. Kumar, R. G. Sonkawade, S. K. Chakarvarti et al., "Study of optical, structural and chemical properties of neutron irradiated PADC film," Vacuum, vol. 86, no. 3, pp. 275-279, 2011.

[21] M. Zaki, "Gamma-induced modification on optical band gap of CR-39 SSNTD,” Brazilian Journal of Physics, vol. 38, no. 4, pp. 558-562, 2008.

[22] E. Moura, A. Ortiz, H. Wiebeck, A. Paula, A. Silva, and L. Silva, "Effects of gamma radiation on commercial food packaging films-study of changes in UV/VIS spectra," Radiation Physics and Chemistry, vol. 71, no. 1-2, pp. 201-204, 2004.

[23] S. Singh and S. Prasher, "The etching and structural studies of gamma irradiated induced effects in CR-39 plastic track recorder," Nuclear Instruments and Methods in Physics Research Section B: Beam Interactions with Materials and Atoms, vol. 222, no. 3-4, pp. 518-524, 2004.

[24] R. C. Abul-Hail, "Optical limiting behavior correlated with the surface and etching time of irradiated nuclear track detector," Journal of Materials Science: Materials in Electronics, vol. 28, no. 3, pp. 2311-2316, 2017.

[25] K. Alfaramawi, M. Al-Rasheedi, M. Baig, and M. Alsalhi, "Solar radiation-induced changes in optical characteristics of PM-355 polymeric films," Radiation Measurements, vol. 86, pp. 49-55, 2016.

[26] M. Zaki, T. M. Hegazy, and D. H. Taha, "Investigation of the effect of a He-Ne laser on the optical properties through etched CN-85 and CR-39 containing alpha tracks," Chinese Journal of Physics, vol. 52, no. 4, pp. 1364-1375, 2014. 